$\begin{array}{ll}\text { le portiQue } & \text { Le Portique } \\ \text { Revue de philosophie et de sciences humaines }\end{array}$

$20 \mid 2007$

Gilles Deleuze et Félix Guattari : Territoires et devenirs

\title{
Deleuze-Guattari et la prise de parole
}

Frédéric Astier

\section{OpenEdition}

\section{Journals}

Édition électronique

URL : http://journals.openedition.org/leportique/1373

DOI : $10.4000 /$ leportique.1373

ISSN : $1777-5280$

\section{Éditeur}

Association "Les Amis du Portique"

Édition imprimée

Date de publication : 15 décembre 2007

ISSN : 1283-8594

\section{Référence électronique}

Frédéric Astier, « Deleuze-Guattari et la prise de parole », Le Portique [En ligne], 20 | 2007, mis en ligne le 07 novembre 2009, consulté le 25 mars 2021. URL : http://journals.openedition.org/leportique/1373 ; DOI : https://doi.org/10.4000/leportique.1373

Ce document a été généré automatiquement le 25 mars 2021.

Tous droits réservés 


\title{
Deleuze-Guattari et la prise de parole
}

\author{
Frédéric Astier
}

1 Traverser la rue de Richelieu par les souterrains de la Bibliothèque nationale pour acheminer d'antiques disques 78tour/minute vers les studios Vivienne du Département de la Phonothèque, le chemin m'était familier, mais un matin, surprise, j'entendis derrière un épais mur de verre la voix de Gilles Deleuze, sur un bruit de fond d'autoroute, amplifié par une salle préfabriquée habituellement destinée aux migrants étrangers (les LOPOFA). Des cassettes audio de quelques étudiants nous étaient parvenues en très grande quantité. Quelques mois plus tard, humble employé, fort de quelques années d'études à l'Université de Paris 8 , je proposais à mes responsables de m'occuper du pointage, et par suite du classement, de l'étiquetage, de la cotation et de la vérification dite technique des durées de ces enregistrements transférés sur disques compacts, et pour cela, il fallut les entendre.

2 Hasard, je me souviens avoir ri en l'écoutant, lorsque de sa voix rauque et caillouteuse, il affirme que le philosophe doit parler au non philosophe, ou encore, que l'idiot de Nicolas de Cuses est un très bon observateur. Je me sentais dans la peau de l'un et de l'autre, c'est peu dire. Et c'est ainsi que des mois durant, je pris note des idées développées dans ces 177 cours, 400 heures - de 1979 à 1987, un travail d'ombre.

Aborder la pensée de Gilles Deleuze, pensais-je, nécessitait des rudiments et un horizon de références, car notre auteur avait beau dire «ne vous inquiétez pas, tout le monde peut comprendre », philosophe ou non, je ne le croyais guère.

4 Son invite cependant fut très efficace - «prenez ce que vous voulez, ce qui vous convient, c'est vous qui voyez »-, elle me permit d'être à l'aise, un certain confort et en même temps, l'obligation de se demander : mais qu'est-ce qui me concerne dans ce qu'il dit?

Le style Deleuze. On peut dire que ses cours lui permettaient d'expérimenter des suites logiques, celles de ses futurs chapitres, mais à une vitesse inversée du brillant professeur. Très lentement, par flux et reflux, il avançait, reculait. Deleuze manifestement suivait des notes, très précises, même si ses références d'éditions, de dates, ou de pages étaient lacunaires. Son cours était expérimentation au sens fort. 
Il entendait si les connexions se réalisaient de temps à autre, les petites lumières des regards.

(Extrait de l'ouvrage Les cours enregistrés de Gilles Deleuze, 1979-1987, Sils-Maria, 2006.) désormais dans le discours philosophique en général, Deleuze et Foucault affirment une rupture avec l'histoire de la pensée et une nouvelle perspective pour la pensée. Cet écart se fonde sur la lecture renouvelée de l'œuvre de Nietzsche. La somme des textes inédits ou manuscrits dont il s'agit de fondre dans l'œuvre de Nietzsche, implique une critique et des renversements probables de sens et d'interprétation des lectures dans la mesure où « Nietzsche a ouvert une blessure dans le langage philosophique » ${ }^{2}$.

Pour Deleuze, toute création, en son propre domaine - pictural, scientifique, philosophique, cinématographique - s'élabore selon une idée et un espace-temps singuliers. Deleuze enseignant multiplie les images de ces espaces-temps lors de ses cours, à travers les différentes rubriques et les points abordés.

Les cours se développent et s'agencent à partir d'une accumulation d'écarts, d'intervalles, entre une succession, une conjonction de rubriques, combinant ainsi une gamme d'intensités pour la pensée. Les intervalles produisent une tension singulière, une béance du sens où se joue son actualité et sa virtualité. Il faut produire des connexions (tant pour l'enseignant que pour l'auditeur) entre des flux, suivant des lignes de fuite. Deleuze emploie le terme de «fluxion " ${ }^{3}$, lorsqu'il s'agit par exemple d'une connexion possible, «révolutionnaire», d'un rapport par tangente entre deux flux, l'un émanant d'un état minoritaire, l'autre d'un flux majoritaire. Une rencontre, une fluxion est toujours possible, probable, entre les rubriques, entre les points des cours. L'auditeur peut relier pour lui-même deux idées, dont l'une, exposée lors du cours, s'apparente à telle autre provenant de son expérience, de sa discipline, de ses recherches. L'auditeur dépasse ainsi le domaine exclusif d'une idée, d'une connaissance ou d'une discipline.

Où donc réside l'inspiration du philosophe lors des cours? Signe-t-elle le moment d'une nouveauté de pensée pour lui? Comment se manifeste-t-elle? Peut-elle être silencieuse, opérant entre les rubriques ou doit-elle être localisée? L'inspiration de Deleuze trouve souffle et style probablement à la suite de telle lecture du paragraphe d'un texte, ou lorsqu'elle se rapporte à la mesure d'un certain degré d'intensité des auditeurs captivés, accompagnant silencieusement le propos, les petites lumières des regards.

9 Une pensée s'appréhende à partir de signes, d'un régime de signes, qu'il s'agit de capter, peu à peu, au fil des séances.

Ces écarts produisent des tensions qui alimenteront pour l'auditeur des compréhensions retardées. Ces effets retardés de la compréhension se réaliseront lors d'un cours, ou bien lors des séances suivantes, ou bien même encore, justement, " entre » les cours, c'est-à-dire hors la salle de cours, en extériorité, par multiplicités.

11 En 1979, l'Université de Paris 8 est transférée de Vincennes à Saint-Denis, Félix Guattari ne participe plus aux séminaires, la double signature Deleuze-Guattari des livres est suspendue. Affaire de style, c'est avec les auditeurs que Deleuze, désormais, entreprend une nouvelle démarche de sa pensée; lors des cours à Saint-Denis, les idées du philosophe s'encrent et s'incarnent selon une proximité et une coexistence différentes : les auditeurs participent davantage à l'élaboration des trames conceptuelles du 
philosophe. Son cours de philosophie, du Lycée à l'Université, Deleuze l'a toujours minutieusement préparé, répété.

La communication intitulée La méthode de dramatisation ${ }^{4}$ nous livre, auditeurs et lecteurs, quelques clefs qui nous permettent de percevoir la perspective générale d'un cours agencé par Deleuze, d'aborder une séance et d'entendre ou sentir le processus nouant les idées au concept.

Nous proposons de suivre le principe, ou le motif suivant : un concept enseigné, élaboré pendant le cours, se double d'un usage direct, l'enseignant utilise le concept pour mener son enseignement. La transmission du sens et la compréhension de tel concept se constituent dans l'usage de ce même concept. Ce mode d'enseignement serait au cœur même de ce qu'il faut entendre par «dimension artiste» de la philosophie de Deleuze, une philosophie enseignée, transmise oralement.

14 L'appréhension d'un concept passe par le positionnement d'un problème, un problème de pensée dont la difficulté est endurée par Deleuze ; si le cours est préparé et répété et donc mis en scène, n'en demeure pas moins un positionnement de problème sur lequel bute le philosophe écrivain. Ce problème, le philosophe le "déterritorialise», le transportant d'une part dans la dimension orale, et d'autre part, en même temps, dans la dimension de l'enseignement.

15 Un jeu de pensée s'élabore entre une « sphère privée » - celle du penseur écrivain, dans la lignée Schopenhauer-Nietzsche - et le cadre de l'enseignement public de la philosophie à l'Université. Deleuze a été un enseignant classique de philosophie morale à l'Université de Lyon. Cette période qui précède son arrivée à Vincennes n'est pas évoquée à la lettre " $\mathrm{P}$ comme professeur » de l'Abécédaire. Les dix années à Vincennes, entre les lendemains de Mai 1968 et le transfert de l'Université à Saint-Denis, font l'objet d'une critique passant par une nécessaire reformulation de ce qu'il attend et entend de son cours de philosophie.

Un cours du 18/11/1986, filmé par Marielle Burkhalter ${ }^{5}$, intitulé: Leibniz comme philosophie baroque ${ }^{6}$.

17 Caméra de face. Un regard scrutant les auditeurs en un mouvement circulaire, un balayage régulier, pendulaire de gauche à droite, se posant rarement au centre. Le buste incliné, une tête de profil, davantage qu'un visage, rarement de face. Le philosophe est bien parmi les auditeurs, certains assis contre le tableau noir à portée de bras, un quart de tour suffit à l'enseignant et sa craie pour l'atteindre. La main gauche ombre le visage et la soutient, replace les lunettes. Puis les mains jointes, le dos penché et droit, la tête s'enfonce dans les épaules.

18 L'index pointé verticalement signale l'attention requise des auditeurs, puis s'oriente vers le schéma du tableau. "Tout point de l'espace est un point de vue possible», conceptualise Deleuze; un point de vue équivaut à un "point d'ouïe»; certains auditeurs sont debouts au seuil de la porte de la salle.

19 Le flux de paroles accélère, le buste s'allonge horizontalement sous la tension d'une posture " animale», le cou disparaît. Puis, "On a fait beaucoup de progrès depuis la dernière fois, mais de façon élémentaire », affirme, rassurant, l'enseignant. L'index pointé vers le plafond précise une attention, puis le bras d'un quart de tour pivote vers le tableau: "Tout point de l'espace est un point de vue possible 監....

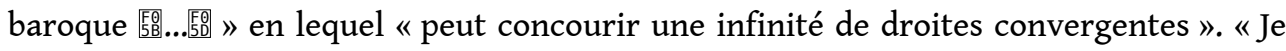

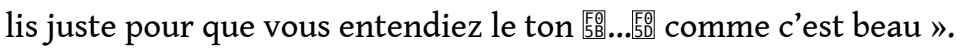


Si Deleuze aborde ensuite la «conscience dramatique» de la perte du centre dans le baroque, le cours lui-même s'émancipe d'un centre, d'une posture de maîtrise de l'enseignant, le buste tourné d'un quart de tour prompt au mouvement vers le tableau noir: "Tout objet est de profil, percevoir, c'est faire une synthèse de profils 嚂L'objectile医. L'objet n'existe que sous ses profils, une série infinie de profils", enchaîne Deleuze.

"Chacun de nous est un point de vue sur la ville», concrétise Deleuze. Le regard en permanence mobile, manifestement scrute les points de vue, «les points d'ouïe » des

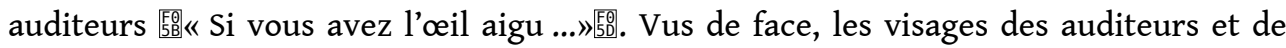
l'enseignant se placent sous les croquis du tableau. Le visage de l'enseignant vu de face et le doigt pointé vers le plafond sont rares et réservés à quelques sentences précises à voix haute.

22 La main droite énumère, soupèse, calme ou avertit, pointe, dirige ou tourbillonne. La main gauche prend le front, replace les lunettes, masque la bouche puis pointe et tend vers le tableau.

La tête orientée à gauche confie ses propos par voix atténuées, énonce une pensée considérée étrange. La tête orientée à droite éclaire, situe une mise en scène, énonce plus abstraitement, ironise ou affirme avec une voix plus forte. Le moment d'une affirmation, le visage et le buste s'abaissent et se figent en une tête aux aguets.

4 À partir de la lecture d'un passage d'une Lettre à Sophie de Leibniz, Deleuze énonce l'espace comme compris dans le point de vue, la ville étant donc un point de vue, que regarder la campagne par une fenêtre équivaut à la campagne elle-même, qu'il n'y a en somme pas de fenêtre mais seulement la campagne. Avec la main gauche qui précise puis nuance, l'affirmation se rythme ; un bref coup d'œil central reprend et corrige. La main droite mesure et signale des degrés. Deleuze aborde ainsi la notion de monologue, monologuer dans la ville, monologuer soi-même la ville par la lecture des «tables d'information » que l'on s'approprie par soi-même, pour soi-même. Enfin il aborde la peinture, la main droite accélère ses mouvements et mime.

Manifestement, Deleuze improvise, il s'adresse à un auditeur puis à un autre, il monologue tout en s'adressant sur le mode du "Je me parle à moi-même", ce qui produit une absence de centre qui capte l'auditeur pour lui enseigner, le renseigner sur le point de vue, le sien.

Deleuze recourt à l'occasion à des expressions familières, grossières : « la ville est gros cerveau », "vous foutez des trucs dans un sac et il en sort un empire ». L'expression soudainement libère un style par une image revécue, banale, quelconque, intime, naturaliste.

27 L'accentuation de la dernière syllabe du dernier mot d'un énoncé signale une affirmation modifiant la posture assise du philosophe, la tête s'abaisse vers l'avant, le buste s'avance et se courbe à l'horizontal.

La posture générale de Deleuze serait bien celle d'un profil en tension vers le tableau. Ce mouvement est l'action essentielle de l'enseignant, telle une " courbure variable " de la posture du philosophe au concept. Deleuze endosse et joue là le rôle d'un "personnage conceptuel " pour enseigner ${ }^{7}$. Si la séance est filmée, Deleuze espère que "quelque chose passera » de son cours grâce à l'image animée. Si le cours est fixé sur une pellicule, afin de renouveler une expérience menée par le groupe Vidéo du 
Département de philosophie, il affirme qu'il captera, à son tour, l'auditoire avec sa propre machine, sa propre toile.

Deleuze engage un corps à corps avec le concept. S'adressant aux auditeurs, le balancier de ses propos s'engage par profil. Or se mettre de profil, c'est «trahir » pour Deleuze et Guattari. Trahir un enseignement dispensé de face, celui du professeur public ${ }^{8}$, c'est permettre le recours au penseur privé afin d'exprimer son propre point de vue. Exprimer son point de vue signifie nécessairement passer par le point de vue d'autrui ${ }^{9}$.

La double multiplicité, des points de vue ou points d'ouïe des auditeurs, à laquelle s'agglomère la multiplicité des références évoquées par Deleuze, élabore une dialogique ${ }^{10}$ circulante, un mouvement dont le tracé conceptuel dégage une énonciation et un voir proches du on, du il impersonnel. Tel concept emprunte une formule inspirée, donc poétique, hésitante, une étrangeté, d'où la compréhension à effet retardé pour l'auditeur. L'énonciation ainsi formulée se disjoint de la parole proférée du maître et d'une appropriation de sens établi. Il s'agit bien là de la ligne de fuite d'une pensée en devenir. Laisser couler une suite de paroles équivaut à un « mur » pour Deleuze, un mur qu'il faut percer ou franchir, ou bien renvoie à un «trou noir » d'où il faut sortir, jaillir.

31 C'est bien avec l'autre - l'auditeur libre, le non philosophe, l'artiste ou l'étudiant provenant d'une autre discipline - par un passage avec autrui, son point de vue ou point d'ouie, que Deleuze expérimente une sortie de ses problèmes de penseur, lors de ses cours à Vincennes-Paris 8 , pour sortir du solipsisme du scribe, du penseur. Entre le philosophe, les auditeurs et la polyphonie des références invoquées, les idées cheminent et le concept s'en saisit, en fonction d'une écoute autant philosophique que non philosophique.

\section{NOTES}

1. . Michel Foucault et Gilles Deleuze veulent rendre à Nietzsche son vrai Visage, in FOUCAULT, Dits et écrits I, 1945-1975, Paris, Gallimard, p. 577.

2. . Ibid.

3. . Le terme de fluxion est énoncé lors du cours du 25/03/1980 enregistré à l'Université de Paris 8.

4. . Gilles DELEUZE, «La méthode de dramatisation", communication du Bulletin de la Société française de Philosophie, $61^{\mathrm{e}}$ année, $\mathrm{n}^{\circ} 3$, juillet-septembre 1967 ; publiée in L'T̂le déserte et autres textes. Textes et entretiens 1953-1974, Paris, Éditions de Minuit, 2002.

5. . Une partie des archives sonores des cours de Deleuze est consultable sur le site La voix de Gilles Deleuze en ligne: http://www.univ-paris8.fr/deleuze/. Afin d'aider les auditeurs dans leurs recherches, nous nous permettons de renvoyer à notre ouvrage: Les Cours enregistrés de Gilles Deleuze, 1979-1987, Mons, Sils Maria, 2006.

6. . Ce cours filmé, intitulé : "Gilles Deleuze : le point de vue : le pli, Leibniz et le baroque », est consultable sur les postes audiovisuels de la Bibliothèque nationale de France, sous la cote VKR-185.

7. . «Et la philosophie ne cesse de faire vivre des personnages conceptuels, de leur donner vie. », in Gilles Deleuze et Félix GUATTARI, Qu'est-ce que la philosophie ?, Paris, Minuit, 1991, p. 61. 


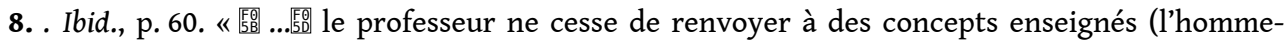
animal raisonnable), tandis que le penseur privé forme un concept avec des forces innées que chacun possède en droit pour son compte (je pense)».

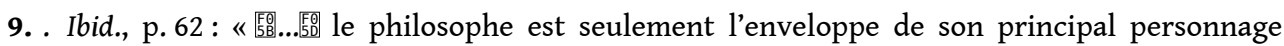

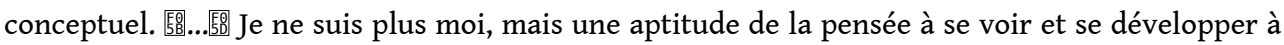
travers un plan qui me traverse en plusieurs endroits. »

10. . Le concept de dialogique (ou polyphonique) de Mikhail Bakhtine constitue sans doute un apport, du moins un renfort conceptuel décisif, transmis à Gilles Deleuze par Félix Guattari. Le cours enregistré offre une multiplicité d'idées, de thèmes philosophiques, de références et de visions, agencé par Gilles Deleuze, selon son art du discours indirect libre.

\section{RÉSUMÉS}

Les enregistrements sonores des cours de Gilles Deleuze à l'Université de Paris 8, 1979-1987 (177 cours, 400 heures) nous transportent, auditeurs et lecteurs, entre les flux et reflux de paroles et les lignes des chapitres du philosophe. C'était le moment d'affûter ou d'élaborer un concept, à partir d'une problématique, abstraite, puis d'une seconde, cette fois-ci concrète, captivant tant l'enseignant que ses apprentis.

Deleuze-Guattari and the 'prise de parole' The sound recordings of Gilles Deleuze's lectures at the University of Paris 8, 1979-1987 (177 courses, 400 hours) transport us, listeners and readers, between flows, backward flows of words and the lines of the chapters of the philosopher. It was the moment to sharpen or to work out a concept, from a abstracted problem, then from a second one this time concrete, captivating the teacher as well as his apprentices.

Deleuze-Guattari und der Wortergriff Die Tonaufnahmen der Vorlesungen von Gilles Deleuze an der Univesität Paris 8 (1979-1987, 177 Vorlesungen, 400 Stunden) lassen uns, als Zuhörer und als Leser, von Ebbe zu Flut zwischen den Aussagen und Zeilen der Kapitel der Philosophen verkehren.

\section{AUTEUR}

\section{FRÉDÉRIC ASTIER}

Frédéric Astier, Doctorant à l'Université de Paris 8, a publié Les Cours enregistrés de Gilles Deleuze, 1979-1987, Sils Maria, 2006. 